\title{
Effect of Natural Compounds on Inhibition of Biofilm Formation of Multi Drug Resistant Staphylococcus aureus and Staphylococcus epidermidis - an in vitro Study
}

\author{
Ankita Agrawal* and Uma Chaudhary \\ Department of Microbiology, Pt. B. D. Sharma PGIMS, Rohtak, Haryana, India \\ *Corresponding author
}

\begin{tabular}{|c|}
\hline Keywords \\
\hline $\begin{array}{l}\text { Biofilm inhibition, } \\
\text { Natural compounds, } \\
\text { Minimum biofilm } \\
\text { inhibitory concentration, } \\
\text { Multi drug resistance, } \\
\text { Staphylococci }\end{array}$ \\
\hline Article Info \\
\hline $\begin{array}{l}\text { Accepted: } \\
26 \text { January } 2018 \\
\text { Available Online: } \\
10 \text { February } 2018\end{array}$ \\
\hline
\end{tabular}

\section{Introduction}

Staphylococcus is known to produce biofilms on host surfaces. Biofilm linked infections are of great concern because biofilm associated bacteria can withstand host immune defences, antibiotics, biocides, and hydrodynamic shear forces. Staphylococcus aureus causes a range of infections, from minor skin infections to pneumonia, bacteraemia, sepsis, meningitis and surgical site infections. Staphylococcus epidermidis is a permanent and ubiquitous
Biofilm formation by Staphylococcus aureus and Staphylococcus epidermidis is a common cause of chronic infection and intravascular device failure. Biofilms are resistant to almost all available drugs rendering them difficult to treat. Thus, there is a need to identify natural compounds which can inhibit biofilm formation. A total of 52 multi drug resistant isolates of Staphylococcus aureus and Staphylococcus epidermidis were isolated from various clinical samples. Isolates were identified by standard microbiological procedures. Biofilm formation was seen by modified tissue culture plate method. The in vitro inhibitory activity of reserpine, eugenol, linoleic acid, curcumin, chitosan and berberine in strong biofilm producing isolates with their minimum biofilm inhibitory concentration (MBIC) was calculated. Biofilm formation was seen in $70.4 \%$ isolates of Staphylococcus epidermidis and $48 \%$ isolates of Staphylococcus aureus. In isolates of Staphylococcus aureus, the maximum activity for biofilm inhibition was observed with reserpine (MBIC $0.0156 \mathrm{mg} / \mathrm{ml}$ ) while in case of Staphylococcus epidermidis with eugenol (MBIC 0.0312 $\mathrm{mg} / \mathrm{ml}$ ). In our study, natural compounds were found effective in vitro for inhibition of biofilm formation in Staphylococcus aureus and Staphylococcus epidermidis with reserpine and eugenol most effective and curcumin least effective. 
antibiotics, silver nanoparticle, paramagnetic iron particles and mechanical methods like flushing, chlorination, and ultraviolet disinfection can be used to combat biofilms (Bose et al., 2011).

Natural compounds of plant extracts like reserpine, berberine, curcumin, eugenol, essential oils (EO) like linoleic acid, and chitosan etc. can inhibit biofilm formation. Reserpine (an alkaloid derived from plant Rouwolfia surpentine) and berberine (an alkaloid isolated from Berberis fremontii) inhibit efflux pumps (Stavri et al., 2007). Linoleic acid (a naturally occurring omega-6 essential fatty acid) is capable of inhibiting autoinducer-2 (Al-2) activity in Escherichia coli $\mathrm{K}-12$ biofilm producing strain. Al-2 is a signal transduction molecule in biofilm (Soni et al., 2008). Significant inhibitory activity of eugenol (oily extract from clover leaf) on MRSA and Methicillin- sensitive Staphylococcus aureus (MSSA) biofilm formation in vitro has been observed (Yadav et al., 2015). Naturally occurring polysaccharide chitosan (partially deacetylated poly $\mathrm{N}$-acetyl glucosamine derived from shells of shrimp and other sea crustaceans) resists biofilm formation by bacteria and fungi like Staphylococcus aureus, Klebsiella pneumoniae, Pseudomonas aeruginosa and Cryptococcus neoformans (Martinez et al., 2010). Curcumin (principal curcuminoid of turmeric) has been shown to inhibit biofilm formation and anti-adhesive activity in Helicobacter pylori (Pattiyathanee et al., 2009). It shows inhibition of quorum sensing hence biofilm development by Staphylococcus aureus, uropathogens such as Escherichia coli, Pseudomonas aeruginosa PAO1, Proteus mirabilis and Serratia marcescens (Packiavathy et al., 2014; Sardi et al., 2017).

The present study was carried out to investigate the biofilm formation in Grampositive aerobic bacteria Staphylococcus aureus and Staphylococcus epidermidis. Biofilm inhibitory effect of natural compounds reserpine, berberine, eugenol, linoleic acid, chitosan and curcumin on strong biofilm producing strains was detected.

\section{Materials and Methods}

The present study was conducted in the Department of Microbiology, Pt. B.D. Sharma Post Graduate Institute of Medical Sciences, Rohtak over a period of one year. A total of 52 multidrug resistant isolate comprising Gram positive bacteria Staphylococcus aureus $(\mathrm{n}=25)$ and Staphylococcus epidermidis $(n=27)$ were isolated from pus, urine, blood and sputum samples by standard conventional microbiological techniques.

\section{Detection of biofilm production by Modified Tissue Culture Plate method (MTCP) (Mathur et al., 2006)}

Isolates from fresh agar plates were inoculated in brain heart infusion (BHI) broth supplemented with $2 \%$ sucrose dispensed $2 \mathrm{ml}$ in test tubes and tubes were incubated at $37^{\circ} \mathrm{C}$ for 24 hours. Then the broth was diluted in the ratio of 1:100 with fresh BHI medium. Two hundred $\mu \mathrm{l}$ of this diluted culture broth was then added to 96 well- flat bottom, nonadherent, non-treated polystyrene tissue culture plates (HiMedia Laboratories, Mumbai, India). Uninoculated broth served as control to check sterility and non-specific binding of the medium while blank well served as control to check the quality of tissue culture plate.

These inoculated tissue culture plates were incubated for 24 hours at $37^{\circ} \mathrm{C}$. After incubation, the contents of the wells were removed by gently tapping the plates. The wells were then washed four times with $0.2 \mathrm{ml}$ of phosphate buffered saline (PBS) to remove planktonic forms. Biofilms which remain 
adhered to the wells were fixed with $2 \%$ sodium acetate for 30 minutes and stained with crystal violet $(0.1 \% \mathrm{w} / \mathrm{v})$ for 30 minutes. Excess stain was rinsed off with distilled water. After drying, the wells were then treated with $200 \mu \mathrm{l}$ ethanol/ acetone (80: 20, $\mathrm{v} / \mathrm{v})$ for $15 \mathrm{~min}$ at room temperature, to evade interference with the stained matter at the liquid- air interface, which is not considered to be indicative of biofilm formation (Nahar et al., 2013). Optical densities (OD) of stained adherent biofilms were then determined by an automated micro ELISA reader at wavelength of $570 \mathrm{~nm}$. These OD values were considered as an index of bacterial adhesion and biofilm formation.

\section{Quality control strains}

American Type Culture Collection (ATCC) strains Staphylococcus epidermidis (ATCC 35984), Pseudomonas aeruginosa (ATCC 27853) and Escherichia coli (ATCC 25922) were used as control strains.

Strains with OD value $>0.24$ were strong, $0.12-0.24$ moderate and $<0.12$ weak biofilm producers. Strong biofilm producing bacteria were tested for effect of natural compounds.

The commercially available compounds reserpine, berberine, eugenol, linoleic acid, chitosan and curcumin were dissolved at a concentration of $4 \mathrm{mg} / \mathrm{ml}$ in dimethyl sulfoxide (DMSO, 10\%v/v) aseptically. Two fold serial dilutions of compounds were made to get concentration ranges from 0.0156 $\mathrm{mg} / \mathrm{ml}$ to $4 \mathrm{mg} / \mathrm{ml}$ (Magesh et al., 2013).

\section{Determination of minimum biofilm inhibitory concentration (MBIC) of natural compounds (Magesh et al., 2013)}

The MBIC for each of the natural compounds were determined using modified tissue culture plate method. The strong biofilm isolates maintained in Brain Heart Infusion agar plate were inoculated into sterile BHI broth and incubated at $37^{\circ} \mathrm{C}$ overnight without shaking. The overnight culture was diluted to $0.5 \mathrm{Mc}$ farland standards in fresh BHI medium.

A 96 well microtitre plate was taken and in each well $100 \mu \mathrm{l}$ of test compound was added to $100 \mu \mathrm{l}$ BHI broth culture. The final concentration of the compounds was ranging from $2 \mathrm{mg} / \mathrm{mL}$ in the first well to 0.0078 $\mathrm{mg} / \mathrm{mL}$ in the ninth well.

Broth inoculum was added in $10^{\text {th }}$ well to see biofilm formation. The "media control" only contained $100 \mu \mathrm{l}$ sterile BHI broth was added in $11^{\text {th }}$ well. Microtitre plate was incubated at $37^{0} \mathrm{C}$ in stationary condition for 24 hours.

After incubation, the contents of the wells were removed by gently tapping the plates. The wells were then washed four times with $0.2 \mathrm{ml}$ of phosphate buffered saline (PBS) to remove planktonic forms.

Biofilms which remain adhered to the wells were fixed with $2 \%$ sodium acetate for 30 minutes and stained with crystal violet $(0.1 \%$ w/v) for 30 minutes. Excess stain was rinsed off with distilled water.

After drying, the wells were then treated with $200 \mu \mathrm{l}$ ethanol/ acetone (80: 20, v/v) for 15 $\mathrm{min}$ at room temperature, to solubilize the dried crystal violet stain which was adherent to any biofilm. Optical densities (OD) of stained adherent biofilms were then determined by an automated micro ELISA reader at wavelength of $570 \mathrm{~nm}$. The inhibitory effect of the compound on biofilm production was calculated by subtracting the media control. The minimum biofilm inhibitory concentration (MBIC) is the concentration of the natural compound at which biofilm formation was reduced to $<0.12$ OD value (Fig. 1). 
Table.1 Minimum biofilm inhibitory concentration (MBIC ranging from $2 \mathrm{mg} / \mathrm{ml}$ to 0.0078 $\mathrm{mg} / \mathrm{ml}$ ) of natural compounds in various strong biofilm forming isolates

\begin{tabular}{|l|l|l|l|l|l|l|}
\hline Organism & $\begin{array}{c}\text { Reserpine } \\
(\mathbf{m g} / \mathbf{m l})\end{array}$ & $\begin{array}{c}\text { Eugenol } \\
(\mathbf{m g} / \mathbf{m l})\end{array}$ & $\begin{array}{c}\text { Curcumin } \\
(\mathbf{m g} / \mathbf{m l})\end{array}$ & $\begin{array}{c}\text { Linoleic acid } \\
(\mathbf{m g} / \mathbf{m l})\end{array}$ & $\begin{array}{l}\text { Chitosan } \\
(\mathbf{m g} / \mathbf{m l})\end{array}$ & $\begin{array}{c}\text { Berberine } \\
(\mathbf{m g} / \mathbf{m l})\end{array}$ \\
\hline Staphylococcus aureus & $\mathbf{0 . 0 1 5 6}$ & 0.0312 & 0.25 & 0.0625 & 0.25 & 0.125 \\
\hline Staphylococcus epidermidis & 0.0625 & $\mathbf{0 . 0 3 1 2}$ & 0.5 & 0.125 & 0.125 & 0.125 \\
\hline
\end{tabular}

Fig.1 Biofilm inhibition by natural compounds

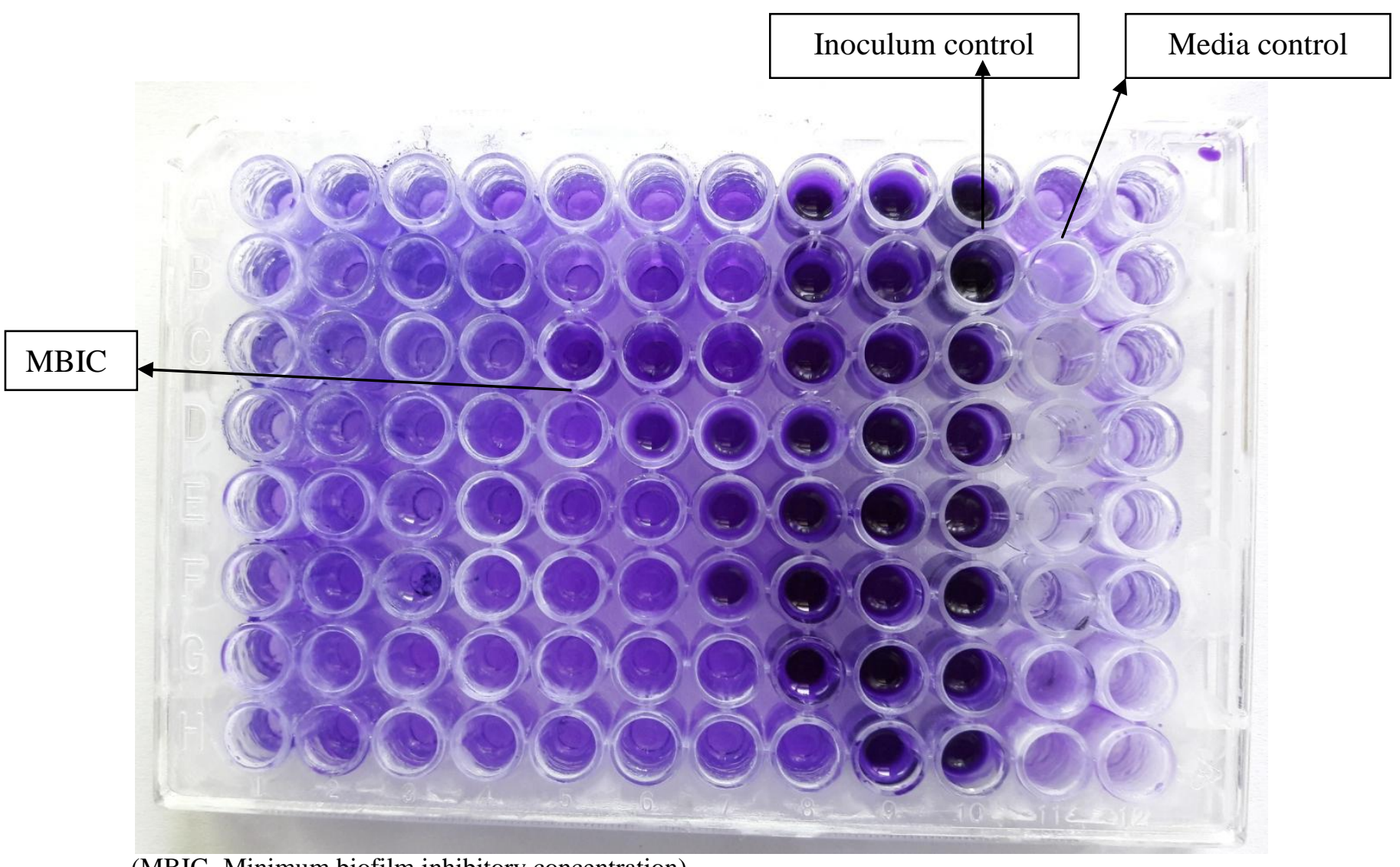

(MBIC- Minimum biofilm inhibitory concentration)

\section{Results and Discussion}

Out of 52 MDR isolates, maximum were obtained from pus $(\mathrm{n}=29)$ followed by blood culture $(n=12)$, urine $(n=8)$, and sputum $(n=3)$ samples. Majority of MDR bacteria were isolated from samples received from indoor $(n=36)$ than outdoor patients $(n=16)$. Biofilm formation was seen in $19(70.4 \%)$ isolates of Staphylococcus epidermidis and 12 (48\%) isolates of Staphylococcus aureus by modified tissue culture plate method. Out of total 31 biofilm forming isolates, 12 (18\%) were strong and $19(32.5 \%)$ moderate biofilm producers. The rest were weak/non biofilm producers.

Biofilm detection can be included as routine diagnostic procedure, so that emergence of resistant isolates can be predicted at the earliest. Prevalence of biofilm formation in different bacteria helps to start empirical 
therapy. In our study, biofilm formation was seen in $59.6 \%$ isolates. Samant et al., found $42.7 \%$ isolates of Staphylococcus species to be biofilm producers (Samant et al., 2012). Jayachandran et al., studied Staphylococci spp. from various clinical isolates. It was observed that $46 \%$ of the isolates were biofilm producers.(Jayachandran et al., 2016) In study done by Apoorva et al., 52.6\% of Staphylococcus aureus were able to produce biofilm (Apurva et al., 2013).

In our study, natural compounds reserpine, berberine, curcumin, eugenol, linoleic acid and chitosan were found effective in inhibition of biofilm formation at MBIC ranging from $2 \mathrm{mg} / \mathrm{ml}$ to $0.0078 \mathrm{mg} / \mathrm{ml}$ in vitro (Table 1). The maximum activity for biofilm inhibition in case of Staphylococcus aureus isolates was for reserpine (MBIC $0.0156 \mathrm{mg} / \mathrm{ml}$ ) while minimum activity was for curcumin and chitosan (MBIC 0.25 $\mathrm{mg} / \mathrm{ml}$ ). The maximum activity for biofilm inhibition in case of Staphylococcus epidermidis isolates was for eugenol (MBIC $0.0312 \mathrm{mg} / \mathrm{ml}$ ) while minimum activity was for curcumin (MBIC $0.5 \mathrm{mg} / \mathrm{ml}$ ).

It was found that MBIC of berberine to inhibit biofilm formation in Staphylococcus epidermidis was $0.125 \mathrm{mg} / \mathrm{ml}$. In contrast, Chu et al., in 2014 found that berberine significantly inhibited biofilm formation in Staphylococcus epidermidis at the concentration of $8 \mathrm{mg} / \mathrm{ml}$. Whereas Wang et $a l$. , found inhibitory effect at concentration of $30-45 \mathrm{mg} / \mathrm{ml}$. In a study by Yadav et al., in 2015, it was observed that eugenol significantly inhibited the growth of Staphylococcus aureus biofilms. At 0.05-0.2 $\mathrm{mg} / \mathrm{ml}$ concentrations, biofilm formation was reduced in eugenol treated isolates, as compared to the control isolates. While in our study, it was inhibited at concentration of $0.0312 \mathrm{mg} / \mathrm{ml}$. Asli et al., observed that chitosan inhibited biofilm formation in Staphylococcus aureus at concentration 16 $\mathrm{mg} / \mathrm{ml}$ while in our study much lower concentration $0.25 \mathrm{mg} / \mathrm{ml}$ was needed. No similar studies were available regarding the reserpine, linoleic acid and curcumin.

Staphylococcus aureus and Staphylococcus epidermidis are the commonly isolated multi drug resistant aerobic bacteria both in indoor and outdoor settings. Persistent infections occur due to biofilm formation by these bacteria. Early identification of infection caused by biofilm producing strains might help to modify treatment and outcome. In our study, natural compounds were found effective in vitro for inhibition of biofilm formation in Staphylococcus aureus and Staphylococcus epidermidis with reserpine and eugenol most effective and curcumin least effective.

\section{Acknowledgments}

Conflict of interest. The authors declare that they have no conflict of interest related to this article.

\section{References}

Apurva, J, D Barate, and M Musaddiq. 2013. "Biofilm forming abilities and antibiotic susceptibility pattern of clinical isolates of Staphylococcus aureus." Ind J App Res. 3:41-44.

Asli A, Brouillette E, Ster C, Ghinet MG, Brzezinski R, Lacasse P, et al., (2017) Antibiofilm and antibacterial effects of specific chitosan molecules on Staphylococcus aureus isolates associated with bovine mastitis. PLoS ONE 12(5): e0176988.

Bose, S, and AK Ghosh. 2011. "Biofilms: A challenge to medical science." J Clin Diag Res. 5:127-30.

Chu, Ming, Ming-bo Zhang, Yan-chen Liu, Jia-rui Kang, Zheng-yun Chu, Kai-lin Yin, Ling-yu Ding, et al., 2016. "Role of berberine in the treatment of methicillin-resistant Staphylococcus aureus infections." Scientific Reports 6 (April):srep24748. 
Jayachandran, Abirami Lakshmy, Sarasa S, Sheila Doris T, Balan K, Sangeetha Vilwanathan, Vanitha Devi E, and Pradeep Balakrishnan. 2016. "Biofilm formation and antibiotic susceptibility pattern among Staphylococcus aureus in a tertiary care hospital in Kanchipuram: An evaluation of screening methods for biofilm formation." Int $\mathbf{J}$ Bioassays. 5 (4):4991-95.

Magesh, H., Arun Kumar, Ayesha Alam, null Priyam, Uma Sekar, Venil N. Sumantran, and Rama Vaidyanathan. 2013. "Identification of natural compounds which inhibit biofilm formation in clinical isolates of Klebsiella pneumoniae.” Indian J Exp Biol. 51 (9):76472.

Martinez, Luis R., Mircea Radu Mihu, George Han, Susana Frases, Radames J. B. Cordero, Arturo Casadevall, Adam J. Friedman, Joel M. Friedman, and Joshua D. Nosanchuk. 2010. "The use of chitosan to damage Cryptococcus neoformans Biofilms." Biomaterials $\quad 31 \quad$ (4):669-79. https://doi.org/10.1016/j.biomaterials.2009.0 9.087.

Mathur, T., S. Singhal, S. Khan, D. J. Upadhyay, T. Fatma, and A. Rattan. 2006. "Detection of biofilm formation among the clinical isolates of Staphylococci: An evaluation of three different screening methods." Indian J Med Microbiol. $24 \quad$ (1):25 https://doi.org/10.4103/0255-0857.19890.

Nahar, Azizun, Shaheda Anwar, and Md Ruhul Amin Miah. 2013. "Association of biofilm formation with antimicrobial resistance among the Acinetobacter species in A tertiary care hospital in Bangladesh." J Med. 14 (1):28-32.

Otto, M. 2009. "Staphylococcus epidermidis - the 'Accidental' pathogen." Nat Rev Microbiol. 7:555-67.

Packiavathy, Issac Abraham Sybiya Vasantha, Selvam Priya, Shunmugiah Karutha Pandian, and Arumugam Veera Ravi. 2014.
"Inhibition of biofilm development of uropathogens by curcumin - an anti-quorum sensing agent from Curcuma longa." Food Chem. 148 (April):453-60.

Pattiyathanee, $\mathrm{P}, \mathrm{R}$ Vilaichone, and $\mathrm{N}$ Chaichanawongsaroj. 2009. "Effect of curcumin on Helicobacter pylori biofilm formation." Afr J Biotechnol.8:5106-15.

Samant, Sharvari, and Chitra Pai. 2012. "Evaluation of different detection methods of biofilm formation in clinical isolates of Staphylococci." Int J Pharm Bio Sci 3 (October):724-33.

Sardi J, Polaquini C, Freires I, Galvao L, Lazarini J, Torrezan G, Regasini L, Rosalen P. 2017. Antibacterial activity of diacetylcurcumin against Staphylococcus aureus results in decreased biofilm and cellular adhesion. $\mathbf{J}$ Med Microbiol. 66(6):816-824.

Soni, Kamlesh A., Palmy Jesudhasan, Martha Cepeda, Kenneth Widmer, G. K. Jayaprakasha, Bhimanagouda S. Patil, Michael E. Hume, and Suresh D. Pillai. 2008. "Identification of ground beef-derived fatty acid inhibitors of autoinducer-2-based cell signaling." J Food Prot. 71 (1):134-38.

Stavri, Michael, Laura J. V. Piddock, and Simon Gibbons. 2007. "Bacterial efflux pump inhibitors from natural sources." $J$. Antimicrob. Chemother. 59 (6):1247-60.

Wang, Xiaoqing, Xiao Yao, Zhen'an Zhu, Tingting Tang, Kerong Dai, Irina Sadovskaya, Sigrid Flahaut, and Said Jabbouri. 2009. "Effect of berberine on Staphylococcus epidermidis biofilm formation." Int J Antimicrob Agents. 34 (1):60-66.

Yadav, Mukesh Kumar, Sung-Won Chae, Gi Jung Im, Jae-Woo Chung, and Jae-Jun Song. 2015. "Eugenol: A phyto-compound effective against Methicillin-Resistant and MethicillinSensitive Staphylococcus aureus clinical strain biofilms." PloS One 10 (3):e0119564.

\section{How to cite this article:}

Ankita Agrawal and Uma Chaudhary. 2018. Effect of Natural Compounds on Inhibition of Biofilm Formation of Multi Drug Resistant Staphylococcus aureus and Staphylococcus epidermidis - an in vitro Study. Int.J.Curr.Microbiol.App.Sci. 7(02): 2921-2926.

doi: https://doi.org/10.20546/ijcmas.2018.702.354 